\title{
Efficiency Optimization in a Microscale Vacuum-Gap Thermionic Energy Converter with High Solar Concentration
}

Yuan Wang ${ }^{1}$ and Shanhe $\mathrm{Su}^{2, *}$

\begin{abstract}
A b stract
The irreversible losses resulting from thermal radiation and the space charge effect have been accepted as the primary mechanisms for vacuum-gap thermal electric converter, which raises the question of how to best combine the two mechanisms to maximize the conversion efficiency. Here, we theoretically analyze the performance of a solar concentrating thermionic energy converter (STEC) with microscale interelectrode space. In the proposed STEC system, both near-field thermal radiation and the space charge effect are considered. By building an energy balance equation, the cathode temperature is determined and numerically solved for given solar irradiance. The influences of the optical concentration, voltage and interelectrode space on the heat flux and conversion efficiency are revealed. It is found that the near-field thermal radiation can be approximated by Stefan-Boltzmann formula with effective emissivity 0.04 for $d>1 \mu m$. By interplaying between the two kinds of irreversible losses, the maximum conversion efficiency and the corresponding interelectrode space of STEC are pointed. Our investigation provides an effective mean to optimal design the interelectrode space of the STEC.
\end{abstract}

Keywords: thermionic emission; space charge effect; near-field thermal radiation; optimal design; maximum efficiency Received: 20 December 2019; Accepted: 4 February 2020

Article type: Research article

\section{Introduction}

Solar-driven solid state energy converters such as thermoelectric generators ${ }^{[1,2]}$ and thermionic emission energy converters (TECs) $)^{[3,4]}$ are a class of important devices for solar energy utilizations. Both the thermoelectric and thermionic generators $^{[5,6]}$ employ electrons as the working fluid and convert directly a part of thermal energy into electrical energy without mechanical motion. ${ }^{[7]}$ Many researchers investigated the performance of a solar-driven thermionic energy converter (STEC) due to its high-power density. Akihisa et al. experimentally found that the emitter with a light trap can effectively improve the energy conversion efficiency of the

${ }^{1}$ Henan Key Laboratory of Electronic Ceramic Materials and Application, Henan University of Engineering, Zhengzhou, 451191, People's Republic of China

${ }^{2}$ Department of Physics, Xiamen University, Xiamen 361005, China

*E-mail: sushanhe@xmu.edu.cn (S. Su)
STEC ${ }^{[8]}$ Wei et al. utilized the illumination of a xenon lamp with a broad spectrum to improve TEC characteristics. ${ }^{[9]}$

In the interelectrode space, the irreversible losses due to the near-field thermal radiation and the space charge effect play an important role in TECs' optimal design. ${ }^{[10]}$ The space charge effect has been thoroughly explored in Refs. [11] and [12] as well. Cesium plasma has been applied in TECs to neutralize the space charge. ${ }^{[13]}$ The method of employing diamond coating with a negative electron affinity has also been provided to increase the emitted current ${ }^{[14,15]}$ Another effective methods to mitigate the space charge effect is to reduce the interelectrode space. ${ }^{[16,17]}$ However, when the gap decreases and becomes comparable with or shorter than the characteristic wavelength of the heat source, near-field radiative heat losses between electrodes become dominated. ${ }^{[18]}$ In addition, when the nanoscale interelectrode is considered, quantum tunneling phenomenon appears. ${ }^{[1,20]}$ Therefore, the optimal design of the 
vacuum gap is of great importance to improve the performance of the STEC. In Ref. [16], the effects of the space charge and near-field radiative heat losses have been considered and the interelectrode gap has been optimized. Starting from Ref. [16], we will establish an energy balance equation to determine the temperature of cathode.

In the present paper, a STEC model consisting of a solar collector and a vacuum thermionic generator with microscale vacuum gap is used to investigate the performance of the hybrid system. The concrete contents of the paper are organized as follows. In Sec. 2, general expressions for the efficiencies of the solar collector, TEC, and STEC system are derived. In Sec. 3, the maximum efficiencies of the STEC system under differently operating conditions are calculated. The effects of interelectrode space, output voltage, and optical concentration on the performance of the system are discussed.

\section{AN IRREVERSIBLE STEC SYSTEM}

The schematic diagram of a STEC system consisting of a solar collector and a TEC is shown in Fig. 1(a), where the collector mainly includes a lens and a wavelength-selective absorber, the TEC mainly consists of a cathode and an anode metal plate with a vacuum gap in the middle. The absorber with the wavelength-selective surface area $A_{s}$ at temperature $T_{s}$ is coupled to the cathode of the TEC with surface area $A_{C}$ at temperature $T_{C}$. The absorber and the cathode are assumed to have the same temperature and area, i.e. $T_{s}=T_{C}$ and $A_{s}=A_{C}$. When the heat leakage losses between the absorber and the ambient obey radiation law, the heat current density released by the absorber and the efficiency of the solar collector can be, respectively, written as ${ }^{[1,21]}$

$$
Q_{h}=C_{o p t} \eta_{o p t} q_{s}-E_{S} \sigma\left(T_{C}^{4}-T_{0}^{4}\right),
$$

and

$$
\eta_{c}=\eta_{o p t}-\frac{E_{s} \sigma\left(T_{C}^{4}-T_{0}^{4}\right)}{C_{o p t} q_{s}},
$$

where the areas of the selective surface and the cathode are assumed to be uniform. $\eta_{o p t}$ is the optical efficiency, $C_{o p t}$ is optical concentration with a unit area of the cathode, $q_{s}$ is the solar irradiance, $E_{s}$ is the effective emissivity of the collector, $T_{0}$ is the ambient temperature, and $\sigma$ is the Stefan-Boltzmann constant.

When the system is operating, and the heat is transferred from the absorber to the cathode of TEC, electrons whose energy are larger than the cathode work function will be emitted out of the cathode surface and transfer to the anode. During this process, the charges between the two electrodes may repel each other and produce an additional energy barrier (ADB) $\varphi_{m}$, as shown in Fig. 1(b), which will dramatically reduce the current density. ${ }^{[22]}$ Fig. 1(b) is the energy diagram of the TEC, where $E_{F, C}\left(E_{F, A}\right)$ is the Fermi level of the cathode (anode), $\Phi_{C}\left(\Phi_{A}\right)$ is the work function of the cathode (anode).
$V$ is the voltage and expressed as $V=\left(E_{F, C}-E_{F, A}\right) / e$. In the flat-band case, $V=\left(\Phi_{C}-\Phi_{A}\right) / e$, where $e$ is the elementary charge ${ }^{[23]}$ The electrons current density that arriving the anode from the cathode and the reversing current density from the anode can be expressed as ${ }^{[16]}$

$$
J_{C}=A_{0} T_{C}^{2} \exp \left(-\frac{\Phi_{C}+\varphi_{m}}{k_{B} T_{C}}\right)=A_{0} T_{C}^{2} \exp \left(-\frac{\psi_{m}}{k_{B} T_{C}}\right),
$$

and

$$
J_{A}=A_{0} T_{A}^{2} \exp \left(-\frac{\psi_{m}-e V}{k_{B} T_{A}}\right),
$$

where $A_{0}$ is the Richardson-Dushmann constant, and $k_{B}$ is the Boltzmann constant. $\varphi_{m}$ can be obtained by using the Langmuir's space-charge theory ${ }^{[24]}$ In our previous work, ${ }^{[2]}$ the effective potential barrier $\psi_{m}$ has been derived in detail, which can be written as

$$
\psi_{m}=\left\{\begin{array}{cc}
\Phi_{C}, & 0<V<V_{S} \\
\Phi_{C}+\gamma_{C m} k_{B} T_{C}, & V_{S}<V<V_{C} \\
\Phi_{A}+e V, & V>V_{C}
\end{array},\right.
$$

where $\gamma_{C m}$ is the dimensionless factor due to the ADB referring to the cathode surface and can be calculated by $\gamma_{C m}=\ln J_{C S} / J_{C}, J_{C S}$ is the saturation current density of cathode, $V_{S}$ and $V_{C}$ are two critical voltages ${ }^{[25]} V_{S}$ is defined as the saturation point. When $V=V_{S}$, the maximum energy barrier occurs just outside the cathode. All emitting electrons from the cathode can arrive in the anode. When $V=V_{C}$, the maximum energy barrier occurs just in front of the anode. The electrons from the cathode need to overcome a decelerating force.

By using Eqs. (3)-(5), the net current density can be expressed as

$$
\begin{gathered}
J=J_{C}-J_{A}=A_{0} T_{C}^{2} \exp \left(-\frac{\psi_{m}}{k_{B} T_{C}}\right)- \\
A_{0} T_{A}^{2} \exp \left(-\frac{\psi_{m}-e V}{k_{B} T_{A}}\right) .
\end{gathered}
$$

The net heat current taken by the thermionic emission electrons from the cathode is defined as

$$
Q_{T}=\left[J \psi_{m}+2 k_{B}\left(T_{C} J_{C}-T_{A} J_{A}\right)\right] / e .
$$

Besides the heat transport taken by the thermionic emission electrons, the radiation heat loss during the electrode space also has a strong influence on the performance of the TEC. Generally, for large interelectrode gaps, the Stefan-Boltzmann law is employed to estimate the propagating-wave heat transfer. ${ }^{[2,27]}$ However, the Stefan Boltzmann law becomes failed, when the spacing is on the order of the characteristic wavelength of thermal radiation (i.e. $\lambda_{T} \approx 2.9 \times 10^{-3} \mathrm{~m} \cdot \mathrm{K} / T_{C}$ ), which given by the Wien 's displacement law. More importantly, the evanescent waves from the two electrodes begin to couple with each other. By using the fluctuational electrodynamics, the contributions of propagating and evanescent modes to the net radiation heat 
transfer can be calculated from the following equations ${ }^{[16,18]}$

$$
\begin{aligned}
Q_{\text {Prop }}= & \frac{1}{\pi^{2}} \int_{0}^{\infty} d \omega\left[\Theta\left(\omega, T_{C}\right)-\Theta\left(\omega, T_{A}\right)\right] \times \\
& \int_{0}^{\omega / c} \sum_{l=s, p} \frac{\left(1-\left|r_{0 \mathrm{C}}^{l}\right|^{2}\right)\left(1-\left|r_{0 \mathrm{~A}}^{l}\right|^{2}\right)}{4\left|1-r_{0 \mathrm{C}}^{l} r_{0 \mathrm{~A}}^{l} \exp \left(-2 d \gamma_{0}\right)\right|^{2}} \beta d \beta,
\end{aligned}
$$

and

$$
\begin{aligned}
Q_{\text {Evan }}= & \frac{1}{\pi^{2}} \int_{0}^{\infty} d \omega\left[\Theta\left(\omega, T_{C}\right)-\Theta\left(\omega, T_{A}\right)\right] \times \\
& \int_{\omega / c}^{\infty} \sum_{l=s, p} \frac{\operatorname{Im}\left(r_{0 \mathrm{C}}^{l}\right) \operatorname{Im}\left(r_{0 \mathrm{~A}}^{l}\right) \exp \left[-2 d \operatorname{Im}\left(\gamma_{0}\right)\right]}{\left|1-r_{0 \mathrm{C}}^{l} r_{0 \mathrm{~A}}^{l} \exp \left[-2 d \operatorname{Im}\left(\gamma_{0}\right)\right]\right|^{2}} \beta d \beta .
\end{aligned}
$$

Here $\Theta(\omega, T)=\hbar \omega /\left[\exp \left(\hbar \omega / k_{B} T\right)-1\right]$ is the mean energy of a Planck oscillator at the angular frequency $\omega . r_{i j}^{l}$ corresponds to the $l$-polarization reflectivity coefficient at the $i$-j interface and $\gamma_{0}=\sqrt{(\omega / c)^{2}-\beta^{2}} \cdot \beta$ is the parallel wavevector. The overall radiation heat losses between the two electrodes are defined as $Q_{L}=Q_{\text {Prop }}+Q_{\text {Evan }}$. By modifying the cathode surface, such as depositing a wide bandgap semiconductor, the heat losses can be compensated.

Using a Drude model for metals, ${ }^{[18]}$ we can write the dielectric function as follow

$$
\varepsilon(\omega)=1-\frac{\sigma_{0} / \tau}{\varepsilon_{0}\left(\omega^{2}+i \omega / \tau\right)},
$$

where $\tau=1 /\left(\alpha T^{2}+\beta T^{3}\right)$ with $\alpha=10^{7} \mathrm{~s}^{-1} \mathrm{~K}^{-2}$ and $\beta=2 \times$ $10^{6} \mathrm{~s}^{-1} \mathrm{~K}^{-3}$, and

$$
\begin{aligned}
\sigma_{0}= & 5.5706 \times 10^{-13} T^{6}-7.33 \times 10^{-9} T^{5}+ \\
& 3.8673 \times 10^{-5} T^{4}-0.1045 T^{3}+153.2994 T^{2}- \\
& 1.1826 \times 10^{5} T+4.1465 \times 10^{7} \mathrm{~S} / \mathrm{m} .
\end{aligned}
$$

The heat taken by the electrons and photons from the cathode can be expressed as

$$
Q_{h}=\left[J \psi_{m}+2 k_{B}\left(T_{C} J_{C}-T_{A} J_{A}\right)\right] / e+Q_{L} .
$$

Based on Eqs. (1) and (12), the energy balance equation for the cathode can be written as

$$
\begin{aligned}
& C_{\text {opt }} \eta_{\text {opt }} q_{s}-E_{S} \sigma\left(T_{C}^{4}-T_{0}^{4}\right)- \\
& \quad\left[J \psi_{m}+2 k_{B}\left(T_{C} J_{C}-T_{A} J_{A}\right)\right] / e-Q_{L}=0 .
\end{aligned}
$$

The power output density and efficiency of the TEC, respectively, given by

$$
P=J V
$$

and

$$
\eta_{T}=\frac{P}{Q_{h}} .
$$

The overall efficiency of the STEC is then determined by

$$
\eta_{s}=\frac{P}{C_{o p t} q_{s}}=\eta_{c} \eta_{t},
$$

which is simply the collector efficiency multiplied by the TEC efficiency.

\section{RESULTS AND DISSCUSSIONS}

By using the data in Table 1 and Eqs. (5), (6) and (13), the curves of the effective potential barrier, cathode temperature and net current density of TEC can be plotted, as shown in Fig. 2. According to Eq. (5), the two critical voltages ( $V_{S}$ and $\left.V_{\mathrm{C}}\right)$ are pointed. For $V<V_{S}\left(V<\Phi_{C}-\Phi_{A}\right)$, the device operates in the saturation region. The cathode temperature keeps constant and $\psi_{m}=\Phi_{C}$. All emitted electrons of the cathode can be collected by the anode. The current density collected by the anode is described by $J_{C S}=A_{0} T_{C}^{2} \exp \left(-\Phi_{C} / k_{B} T_{C}\right)$. And the reverse current is written as $J_{A}=A_{0} T_{A}^{2} \exp \left[-\left(\Phi_{C}-e V\right) / k_{B} T_{A}\right]$. For $V_{S}<V<V_{C}$, the device operates at the space charge limited region. The accumulation of electrons in vacuum leads to an ADB. The current density $J_{C}$ is determined by the electrons which can overcome the ADB. The maximum energy barrier for the reverse electrons is $\psi_{m}-V>\Phi_{A}$. For $V>V_{C}$, the electrons in the interelectrode moving to the anode space experience a decelerating process. The maximum energy barrier appears in front of the anode surface. In this case, the reverse current is $J_{A}=A_{0} T_{A}^{2} \exp \left(-\Phi_{A} / k_{B} T_{A}\right)$.

Table 1. Parameters used in the model of a STEC

\begin{tabular}{lc}
\hline \multicolumn{1}{c}{ Parameter } & Value \\
\hline$e(\mathrm{C})$ & $1.6 \times 10^{-19}$ \\
Solar irradiance, $q_{S}\left(\mathrm{~W} / \mathrm{cm}^{2}\right)$ & 0.1 \\
Optical concentration, $C_{\mathrm{opt}}$ & 100 \\
Optical efficiency, $\eta_{\mathrm{opt}}$ & 0.9 \\
Thermal emissivity, $E_{S}$ & 0.05 \\
Stefan-Boltzmann constant, $\sigma\left(\mathrm{W} / \mathrm{cm}^{2} \mathrm{~K}^{4}\right)$ & $5.67 \times 10^{-12}$ \\
Richardson-Dushmann constant, $A_{0}\left(\mathrm{~A} / \mathrm{cm}^{2} \mathrm{~K}^{2}\right)$ & 120 \\
Work function of cathode, $\Phi_{C}(\mathrm{eV})$ & 2 \\
Bolzmann' constant, $k_{B}(\mathrm{~J} / \mathrm{K})$ & $1.38 \times 10^{-23}$ \\
Temperature of the ambience, $T_{0}(K)$ & 300 \\
\hline
\end{tabular}

Fig. 3 gives the $J-V$ characteristic curves for several values of interelectrode space. For $d=0.5 \mu \mathrm{m}$, both the opencircuit voltage and saturation current are restrained. Because, the cathode temperature is suppressed by the enormous nearfield radiation. For $d=1 \mu \mathrm{m}$, two special voltages $V_{S}$ and $V_{C}$ are identical, which indicates that the space charge effect can be negligible for $d<1 \mu \mathrm{m}$. When $d \geqslant 1 \mu \mathrm{m}$, Eq. (5) indicates that the energy barrier is independent to the interelectrode space for $V \leqslant V_{S}$ or $V \geqslant V_{C}$. In addition, the radiation heat transfer between the electrodes can be estimated by the StefanBoltzmann formula which is also independent to the interelectrode space. Thus, the saturation current density and the open-circuit voltage are invariable. For $V_{s}<V<V_{C}$, TEC operates in space charge limited region and the increasing gap enhances the energy barrier during the electrodes, resulting in the suppressing current density.

To evaluate the performance of the STEC, the curves of the efficiency varying with the operating voltage for different interelectrode spaces can be plotted by Eq. (16) and the data in Fig. 3, as shown in Fig. 4. The maximum efficiencies of the 
STEC are marked for several interelectrode spaces. The efficiency is small at large gaps and small gaps and reaches the maximum at gaps on the order of a micron. For $d=1 \mu \mathrm{m}$ and $d=3 \mu \mathrm{m}$, the maximum efficiencies are equal to $30.6 \%$ and $30.4 \%$, respectively, which are much the same. Following Ref. [16], an optimal gap range (a few microns) can be determined, where the efficiency is at least 0.9 of the maximum

The effects of the interelectrode space on the performance are shown in Fig. 5(a), where the voltage is optimized. The curves of the radiation heat loss (black line) and cathode temperature (blue line) are also plotted as shown in Fig. 5(b). As the irreversible loss results from the space charge effect at the large gaps and the near-field radiation at small gaps, the efficiency of the TEC firstly increases and then decreases. The maximum efficiency of the TEC achieves $41.2 \%$. Meanwhile, the increasing gap arouses the increasing cathode temperature, as the black line shown in Fig. 5(b). And the efficiency of the collector monotonously decreases. Using Eq. (16), we can calculate the maximum efficiency of the STEC (30.6\% with $d=1.5 \mu \mathrm{m})$. In Fig. 5(b), a red line is plotted by using the Stefan-Boltzmann formula $\varepsilon_{e f f} \sigma\left(T_{C}^{4}-T_{A}^{4}\right)$ with $\varepsilon_{e f f}=0.04$. It is can be seen that, the red line coincides with black line at large gaps, which indicates that the radiation loss during the TEC can be approximated by the Stefan-Boltzmann formula for $d>1 \mu \mathrm{m}$.

The influences of the anode temperature on the efficiency of the STEC are showed in Fig. 6. The efficiency can be enhanced by reducing the anode temperature. The current density of the anode is relatively low $\left(0.24 \mathrm{~A} / \mathrm{cm}^{2}\right.$ for $T_{A}=700 \mathrm{~K}$ ), which indicates that the anode temperature mainly affects the radiation loss during the electrodes, so that the cathode temperature and efficiency of the TEC decrease with the decreasing anode temperature. However, the efficiency of the collector increases based on Eq. (2). As a result, the maximum efficiency of the STEC increases with the decrease of the anode temperature, as shown in Fig. 6.

In Fig. 7, the efficiencies of the collector, TEC and STEC are of monotonically increasing functions of the optical concentration. From Eq. (13), the larger the optical concentration is, the higher cathode temperature, resulting in the increasing of heat losses. It should be pointed that, when the optical concentration is large enough, the cathode temperature tends to be invariable. As a result, the increase rates of the efficiencies decrease.

\section{CONCLUSIONS}

With the help of the model of STEC, the energy balance equation of the cathode of the STEC is derived and used to determine cathode temperature. Due to the interplay irreversible losses between the space charge effect and the nearfiled thermal radiation, the efficiency of the hybrid system is taken as an objective function and is maximized. The output voltage, interelectrode space, and optical concentration on the efficiency of the system are discussed in detail. The optimal values of the main parameters are determined. For $d>1 \mu \mathrm{m}$, the approximated Stefan-Boltzmann formula is given to describe the propagating-wave heat flux. The results obtained will provide some theoretical bases for the reasonable choice of interelectrode space and best operation of practical STEC systems.

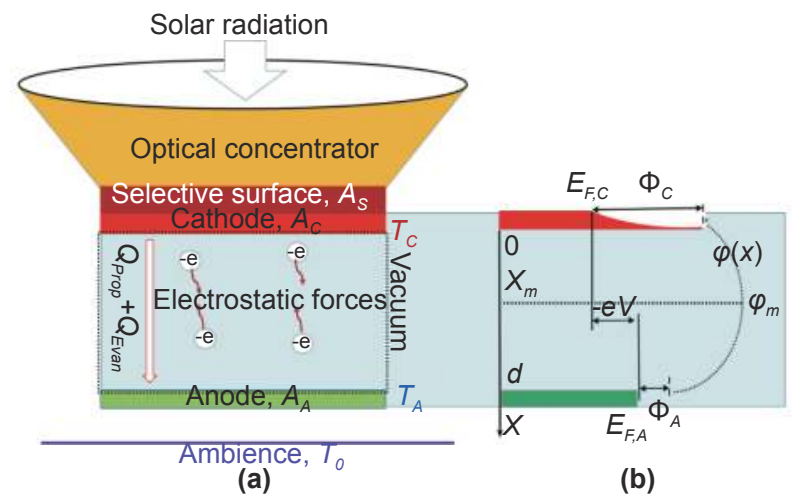

Fig. 1 (a) Schematic diagram of the solar concentrating thermionic energy converter system illustrating its heat loss and electron transport mechanisms. (b) Energy diagram showing the work functions and energy barriers for both the cathode and anode.

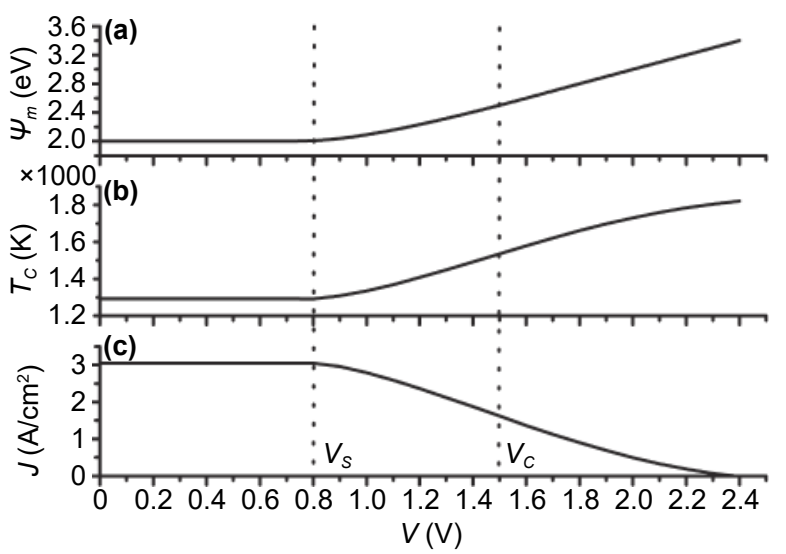

Fig. 2 (a) The effective potential barrier $\psi_{m}$, (b) the cathode temperature $T_{C}$, and (c) the net current density $J$ as a function of output voltage for $d=5 \mu \mathrm{m}$, where $V_{s}$ and $V_{C}$ are two critical voltages defined in Eq. (5).

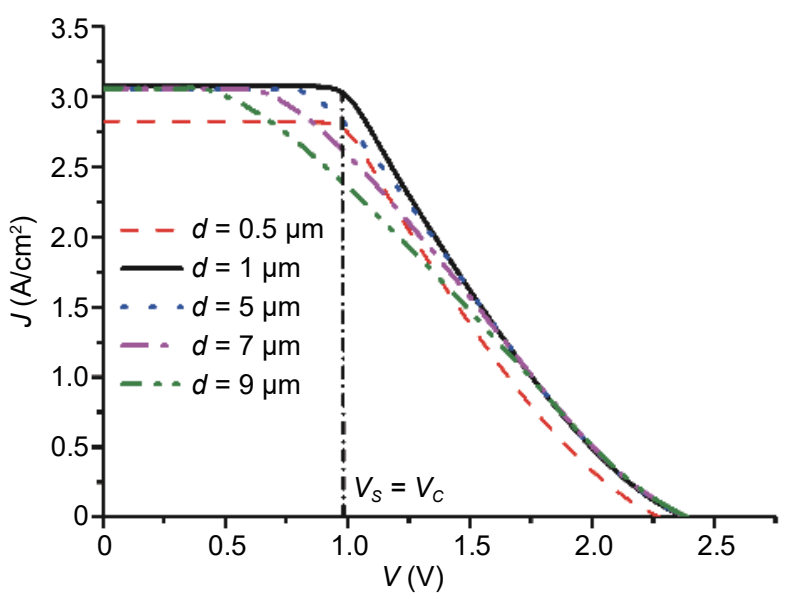

Fig. $3 J-V$ characteristics for different interelectrode spaces. 


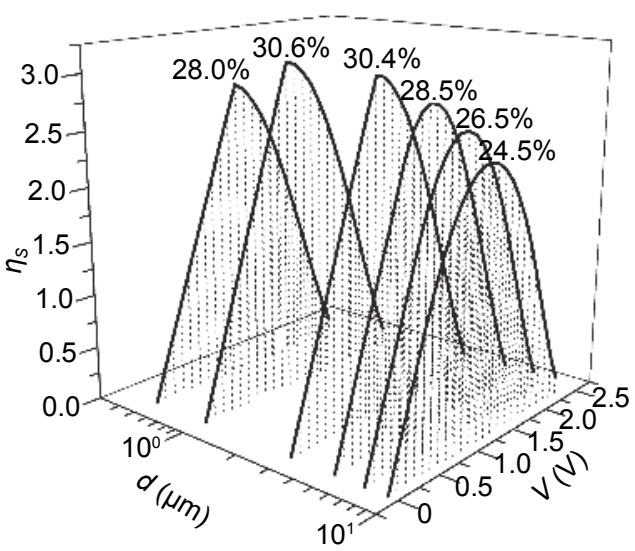

Fig. 4 The efficiency of the STEC as a function of voltage for several values of interelectrode space.
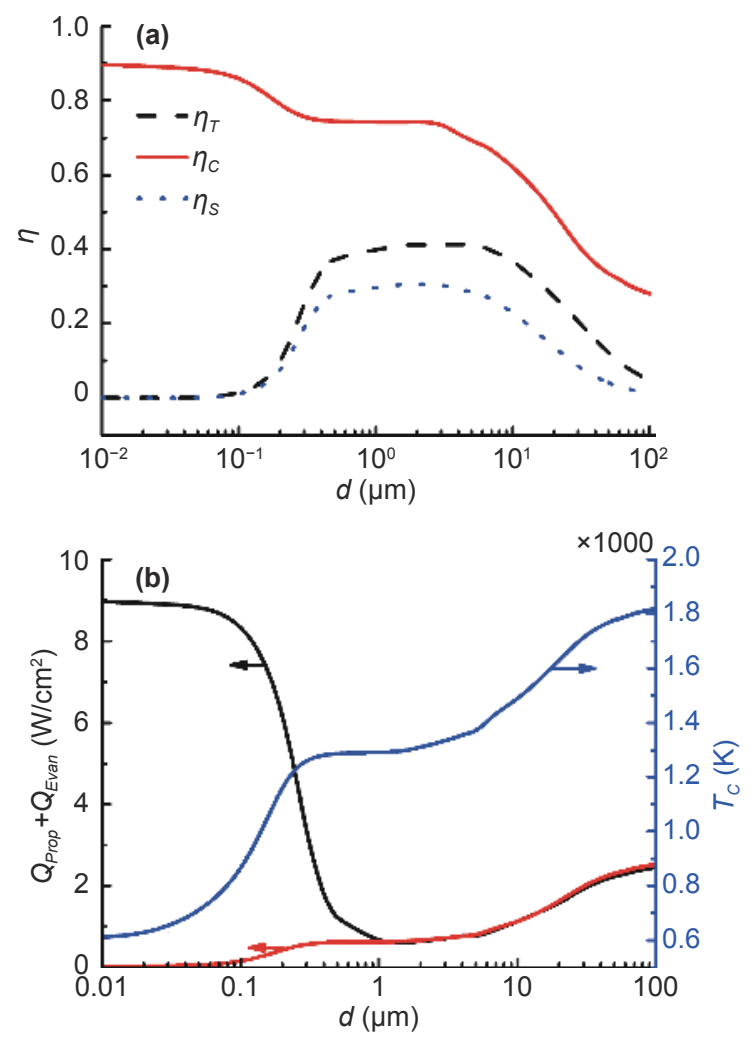

Fig. 5 The effect of the interelectrode space on the (a) efficiency, (b) radiation heat loss, and cathode temperature.

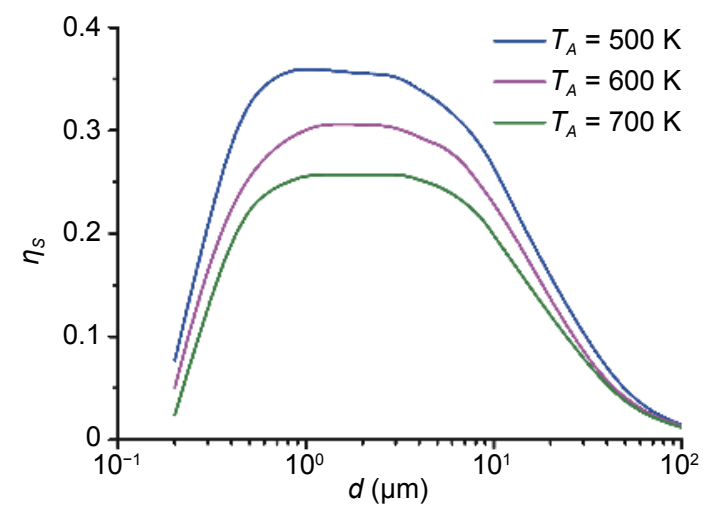

Fig. 6 The influence of the anode temperature on the efficiency of the STEC.

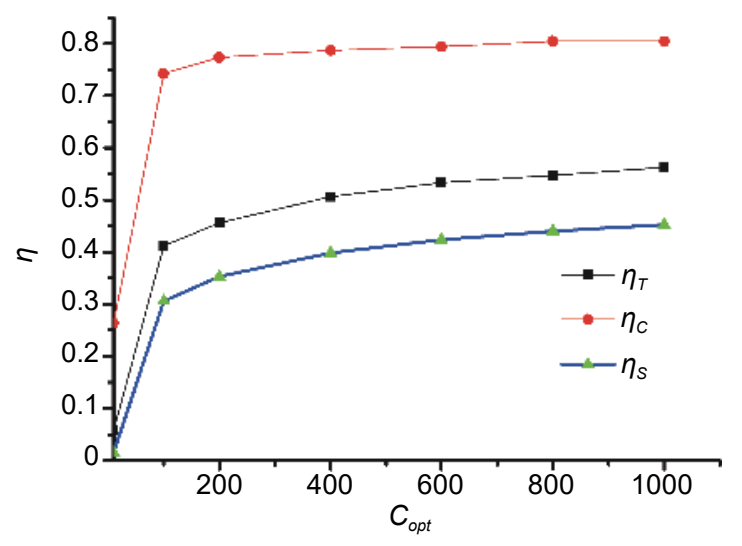

Fig. 7 The influence of optical concentration on the efficiencies of the collector, TEC, and STEC.

\section{Acknowledgments}

This work has been supported by the National Natural Science Foundation (No. 11747036), People's Republic of China.

\section{Supporting information}

Not applicable

\section{Conflict of interest}

There are no conflicts to declare.

\section{References}

[1] D. Kraemer, B. Poudel, H. P. Feng, J. C. Caylor, B. Yu, X. Yan, Y. Ma, X. Wang, D. Wang, A. Muto, K. McEnaney, M. Chiesa, Z. Ren and G. Chen, Nat Mater., 2011, 10, 532-538.

[2] J. C. Chen, J. Appl. Phys., 1996, 79, 2717-2721.

[3] L. Chen, Z. Ding and F. Sun, J. Appl. Phys., 2010, 107, 104507.

[4] J. W. Schwede, I. Bargatin, D. C. Riley, B. E. Hardin, S. J. Rosenthal, Y. Sun, F. Schmitt, P. Pianetta, R. T. Howe, Z. X. Shen and N. A. Melosh, Nat Mater, 2010, 9, 762-767.

[5] G. N. Hatsopoulos and E. P. Gyftopoulos. Thermionic energy conversion. Volume I. Processes and devices. Massachusetts Institute of Technology Press, Cambridge, MA (United States), United States, 1973.

[6] D. M. Rowe. CRC handbook of thermoelectrics. CRC press 1995.

[7] G. Mahan and L. Woods, Phys. Rev. Lett., 1998, 80, 4016-4019.

[8] A. Ogino, T. Muramatsu and M. Kando, Jpn. J. Appl. Phys., 2004, 43, 309-314.

[9] W. Zheng, A. Ogino and M. Kando, Jpn. J. Appl. Phys., 2000, 39, 2816-2824.

[10] A. H. Khoshaman and A. Nojeh, J. Appl. Phys., 2016, 119, 044902 .

[11] G. Segev, D. Weisman, Y. Rosenwaks and A. Kribus, Appl. Phys. Lett., 2015, 107, 013908.

[12] E. M. Hollmann, J. H. Yu, R. P. Doerner, D. Nishijima and R. P. Seraydarian, J. Appl. Phys., 2015, 118, 103302.

[13] D. V. Paramonov and M. S. El-Genk, Energ. Convers. Manage., 1997, 38, 533-549.

[14] J. R. Smith, G. L. Bilbro, R. J. Nemanich, J. Vac. Sci. Technol. B, 2009, 27, 1132-1141.

[15] J. R. Smith, G. L. Bilbro and R. J. Nemanich, Phys. Rev. B., 2007, 76, 245327.

[16] J. H. Lee, I. Bargatin, N. A. Melosh and R. T. Howe, Appl. Phys. 
Lett., 2012, 100, 173904.

[17] Y. Wang, S. Su, B. Lin and J. Chen, J. Appl. Phys., 2013, 114, 053502.

[18] S. Basu, Z. M. Zhang and C. J. Fu, Int. J. Energ. Res., 2009, 33, 1203-1232.

[19] M. F. O. Dwyer, T. E. Humphrey, R. A. Lewis and C. Zhang, J. Phys. D: Appl. Phys., 2006, 39, 4153.

[20] M. F. O. Dwyer, T. E. Humphrey, R. A. Lewis and C. Zhang, J. Phys. D: Appl. Phys., 2009, 42, 035417.

[21] G. Chen, J. Appl. Phys., 2011, 109, 104908.

[22] S. Su, Y. Wang, T. Liu, G. Su and J. Chen, Sol. Energ. Mat. Sol. C., 2014, 121, 137-143.

[23] C. Feng, Y. Zhang, Y. Qian, Z. Wang, J. Liu, B. Chang, F. Shi and
G. Jiao, Opt. Commun., 2018, 413, 1-7.

[24] I. Langmuir, Phys. Rev., 1923, 21, 419-435.

[25] G. N. Hatsopoulos and E. P. Gyftopoulos. Thermionic energy conversion. Volume II. Theory, technology, and application. MIT Press, Cambridge, MA, United States, 1979.

[26] S. J. Liang and L. K. Ang, Phys. Rev. Appl., 2015, 3, 014002.

[27] C. Huang, Y. Pan, Y. Wang, G. Su and J. Chen, Energ. Convers. Manage., 2016, 121, 186-193.

Publisher's Note: Engineered Science Publisher remains neutral with regard to jurisdictional claims in published maps and institutional affiliations. 